\title{
La sociedad, sus acciones y el cambio climático
}

\author{
The unfolding of society and climate change
}

\author{
Hernán Burbano-Orjuela ${ }^{a *}$ \\ ${ }^{a}$ Centro de Investigación en Ciencias Agrarias, Facultad de Ciencias Agrícolas, \\ Universidad de Nariño, Torobajo, Pasto, Colombia. \\ *Autor de correspondencia: hernan.burbano@googlemail.com
}

\section{E D I T O R I A L}

Humans and nature have such a tight relation that due to its very intimate character, sometimes escapes to our sight. The rise of civilizations depended upon natural resources and services, and this dependence will continue in the future, as humans harness and shape nature itself. The systematic description and progressive understanding of the natural world have allowed the ascent and development of the human condition as part of the natural world, which is at the same time source of different natural resources, and subject of study and inspiration of both natural sciences and humanities. Thus, the natural world is the stage where human beings develop their personalities and reach their dreams.

In spite of the original continuum between humans and nature, the exponential increment of human population, in conjunction with an economic system built to satisfy an increasing demand, have broken this original harmony and created a big problem due to the finite character of natural resources. The necessity to produce goods and services under an economic model that favors profitability instead of sustainability, has produced social imbalances that led to injustice, poverty and marginalization. Climate change is one of the major consequences of this model of development, a phenomenon that threatens biodiversity and human survival. To face climate change, it is necessary to build a new society based on global ethical principles, which hand in hand with scientific developments could reduce detrimental anthropogenic effects. In doing so, humans will be obliged to radically change their developmental models and life style.

In the following paragraphs I will present some elements that contribute to the discussion and analysis of the unfolding of society in the context of climate change.

Our planet works as a unique self-regulated system composed by physical, chemical, biological and human elements. Those elements interact with each other through complex fluxes of information at different temporal and spatial scales.

The unfolding of societies was fueled and occurred concurrently with the exploitation of natural resources. Thus, anthropogenic pressure exerted on fine regulated ecosystems, created imbalances that in some cases lead to the collapse of particular societies. The onset of agriculture in the Neolithic and its subsequent technological innovations led to an increase in population and to a restructuring of social organization. Much more recently the Industrial Revolution augmented the level of natural resources exploitation, which resulted in further natural imbalances. It is worthwhile to mention that the climate has been interlinked with human prehistory and history. However, while life on earth, and consequently societies, are constrained by cyclic ecological laws, our economic models trigger linear transformations.

Culture can be understood as an intermediary between humans and their surroundings, which shapes our perception of the natural by instilling "meaning networks". Those networks allow humans to recognize themselves and their rationality as part of the natural world, and trigger a change that leads to respect both nature and fellow human beings.

Although cultural and technological developments allow humans to adapt to an ever changing nature, human and economic welfare mainly depend on natural services provided by ecosystems.

At the end of the $20^{\text {th }}$ century new social problems arose. Their very challenging nature would require that humans take a new attitude towards nature, which based on new social values, will permit an integral improvement on quality of life.

Nowadays, climate change is the principal problem that global society is facing. It results in an average increase of global temperature trigger by the anthropogenic increase of greenhouse gases such as $\mathrm{CO}_{2}, \mathrm{CH}_{4}$ and $\mathrm{N}_{2} \mathrm{O}$. If this challenge is not approached appropriately at a global scale, the level and consequences of climate change will be even more detrimental. For this reason, internationally organizations have put forward sustainable proposals that could change agricultural systems, integrate resilience and productivity, fight poverty and famine, protect the planet, foster personal prosperity, and, overall, counteract climate change.

There is today and antagonisms between the self-regulatory and millennial action of nature and the actions taken by society to reach its ever increasing demands. The fact that the current geological era was named Anthropocene reveals the impact of the very deleterious effects generated by human activity, which challenges our planet viability and therefore human welfare.

Global population has grown disproportionately under a model that is unsustainable. Consequently, the environmental challenge is a social problem that will require global societal engagement. Since there is an indissoluble union between social and biotic factors, the future of humanity relies in this symbiosis. The roadmap is however difficult, as we would need to modify our worldview through a long-term educative action that change present cultural values. 


\section{EDITORIAL}

La relación entre los seres humanos y la naturaleza es una realidad tan evidente que, paradójicamente, en ocasiones no se ve. Las civilizaciones requirieron de los servicios que presta la naturaleza y a futuro continuará esa dependencia, de unos seres inteligentes que de todas maneras también son naturaleza. Ese gran laboratorio que es el mundo natural y que poco a poco se ha venido develando, ha permitido que el planeta sea el gran hogar o la Tierra Patria para que florezca la condición humana, con una naturaleza que, a la vez que es una incógnita permanente para la inteligencia, es el proveedor de hábitat, alimento, recursos diversos, riqueza escénica, donde se cumplen los sueños y el ascenso de las personas.

No obstante lo anterior, la sociedad mundial vive hoy un grave problema, derivado del crecimiento inusitado de un sistema económico que tiene los límites insuperables del tamaño del planeta y de sus recursos, para satisfacer la demanda de bienes y servicios de una población que ya supera los 7.000 millones de habitantes. Esto significa producir más a costa de unos recursos muy agotados, con un aparato productivo que solo busca la rentabilidad y que así genera desajustes sociales -injusticia, pobreza, marginamiento- y que compromete el futuro de las nuevas generaciones. Este patrón de manejo y desarrollo ha conducido a una serie de disfunciones que se evidencian hoy en el cambio climático, fenómeno complejo que cada vez más pone en peligro la continuidad de toda la vida en la Tierra. Esto demandará cambios profundos en el estilo de vida de las personas, acompañado de un despertar en la ética de esta civilización, que la conduzca, sirviéndose con mesura del progreso científico a minimizar esa visión antropocéntrica que podría conducir al colapso.

Los referentes y planteamientos que se presentan a continuación, buscan destacar, en líneas generales, algunos de los elementos que contribuyen al problema objeto de análisis.

La Tierra funciona como un sistema único y auto-regulado, integrado por componentes físicos, químicos, biológicos y humanos. Con interacciones y flujos complejos de información entre sus partes y con gran variabilidad en sus múltiples escalas de espacio y tiempo.

El avance de las sociedades estuvo unido a la integración de los recursos naturales, por eso, cuando los seres humanos desintegraron la trama de la vida, por presión sobre los recursos, sucedieron episodios difíciles que, en ocasiones llevaron al colapso. El inicio de la agricultura en el Neolítico condujo al incremento de la población y la provisión de alimentos, con innovaciones tecnológicas y cambios en la organización social. Más modernamente, los problemas de los recursos naturales en el planeta se empezaron a acentuar con el despegue de la Revolución Industrial. Cabe destacar que el clima siempre estuvo ligado a la historia de la humanidad, que está sometida a las leyes de la ecología, aunque, mientras la economía realiza transformaciones lineales, las de la naturaleza son cíclicas.

Sin embargo, la humanidad desarrolló la cultura, que actúa como intermediario entre los humanos y su entorno y que hace que la percepción de lo natural esté mediada por "redes de significado" y que permite que los humanos se reconozcan como naturaleza y que acepten que la razón no es superior a la naturaleza sino parte de ella, con un cambio de actitud que conlleva el respeto por las personas y por la naturaleza.

Aunque se han desarrollado respuestas tecnológicas y culturales para adaptación al medio natural y a sus fluctuaciones, el bienestar humano y el de la economía dependen esencialmente del flujo de servicios provistos por los ecosistemas naturales. A finales del siglo XX salieron a flote en el mundo muchos problemas sociales que exigían nuevos relacionamientos de los humanos con la naturaleza, apoyados en nuevos valores y tendencias para mejorar la calidad de vida de una manera integral. Al presente, el cambio climático es el principal problema ambiental que enfrenta la sociedad mundial y que trae consigo el incremento de la temperatura media planetaria, como consecuencia del aumento -de origen antrópico- de los GEI, sobre todo $\mathrm{CO}_{2}, \mathrm{CH}_{4}$ y $\mathrm{N}_{2} \mathrm{O}$, fenómeno que irá creciendo si el mundo no encara unido este desafío. Por eso, organismos y propuestas internacionales propenden por un desarrollo sostenible que cambie el enfoque de los sistemas agroalimentarios que amiguen la resiliencia y la productividad, que busquen eliminar la pobreza y el hambre, protejan al planeta y garanticen la prosperidad de todas las personas, en contravía del cambio climático.

En el planeta hay contraposición entre las exigencias de una sociedad empeñada en conseguir solo éxitos económicos, y la acción generosa, callada y milenaria de la naturaleza. La intensidad de los cambios en el planeta que han llegado a configurar una época geológica sui generis, el Antropoceno, revela la realidad de cambios tan nocivos impulsados por los seres humanos que se evidencia en el cambio climático que compromete cada vez más la viabilidad del planeta y el bienestar de las personas. La población mundial ha crecido exageradamente y con hábitos insostenibles. Por eso, el problema ambiental es social y requiere la participación activa de todos, tomando en cuenta que en el planeta hay una unión indisoluble entre lo social y lo biótico, simbiosis donde los seres humanos se juegan la vida. Tomar ese camino no es fácil, porque significa hacer un giro que cambie el imaginario de las personas a través de una acción educativa a largo plazo que tiene que tocar los patrones culturales vigentes. 